\title{
Stabilization in a two-species chemotaxis system with a logistic source
}

\author{
J I Tello and M Winkler
}

\begin{abstract}
We study a system of three partial differential equations modelling the spatiotemporal behaviour of two competitive populations of biological species both of which are attracted chemotactically by the same signal substance. More precisely, we consider the initial-boundary value problem for

$\begin{cases}u_{t}=d_{1} \Delta u-\chi_{1} \nabla \cdot(u \nabla w)+\mu_{1} u\left(1-u-a_{1} v\right), & x \in \Omega, t>0 \\ v_{t}=d_{2} \Delta v-\chi_{2} \nabla \cdot(v \nabla w)+\mu_{2} v\left(1-a_{2} u-v\right), & x \in \Omega, t>0 \\ -\Delta w+\lambda w=u+v, & x \in \Omega, t>0\end{cases}$

under homogeneous Neumann boundary conditions in a bounded domain $\Omega \subset \mathbb{R}^{n}, n \geqslant 1$, with smooth boundary.

When $0 \leqslant a_{1}<1$ and $0 \leqslant a_{2}<1$, this system possesses a uniquely determined spatially homogeneous positive equilibrium $\left(u^{\star}, v^{\star}\right)$. We show that given any such $a_{1}$ and $a_{2}$ and any positive diffusivities $d_{1}$ and $d_{2}$ and crossdiffusivities $\chi_{1}$ and $\chi_{2}$, this steady state is globally asymptotically stable within a certain nonempty range of the logistic growth coefficients $\mu_{1}$ and $\mu_{2}$.
\end{abstract}

\section{Introduction}

We consider two competitive populations of biological species which are attracted by the same chemical stimulus. All individuals move according to random diffusion and chemotaxis, and moreover we assume that both populations reproduce themselves, and mutually compete with the other, according to the classical Lotka-Volterra kinetics [12]. Corresponding to standard 
modelling approaches [12], the respective population densities $u=u(x, t)$ and $v=v(x, t)$ can then, after renormalization, be described by

$$
u_{t}=\underbrace{d_{1} \Delta u}_{\text {diffusion }}-\underbrace{\chi_{1} \nabla \cdot(u \nabla w)}_{\text {chemotaxis }}+\underbrace{\mu_{1} u\left(1-u-a_{1} v\right)}_{\text {proliferation and competition }}
$$

and

$$
v_{t}=\underbrace{d_{2} \Delta v}_{\text {diffusion }}-\underbrace{\chi_{2} \nabla \cdot(v \nabla w)}_{\text {chemotaxis }}+\underbrace{\mu_{2} v\left(1-a_{2} u-v\right)}_{\text {proliferation and competition }}
$$

with parameters $d_{1}, d_{2}, \chi_{1}, \chi_{2}, \mu_{1}, \mu_{2}, a_{1}$ and $a_{2}$, where $w=w(x, t)$ denotes the concentration of the chemoattractant. In numerous biologically relevant processes, the latter signal substance is produced by the cells themselves (see [6] for details), and then its evolution is essentially governed by a parabolic equation of the form

$$
\varepsilon w_{t}=\underbrace{d_{w} \Delta w}_{\text {diffusion }}-\underbrace{\tau w}_{\text {degradation }}+\underbrace{g(u, v)}_{\text {production }}
$$

with positive constants $\varepsilon, d_{w}$ and $\tau$ and a production term $g$ which, in extension to the situation in the classical approach by Keller and Segel [9], depends on $u$ and $v$ now. Under the additional assumption that chemicals diffuse significantly faster than cells, a commonly used mathematically convenient simplification of equation $(0.1)$ is given by

$$
0=d_{w} \Delta w-\tau w+g(u, v)
$$

(see for instance [8]). If we finally assume that both cell types produce the chemical at the same constant rate, without any saturation effects, we are led to considering the system

$$
\left\{\begin{array}{lc}
u_{t}=d_{1} \Delta u-\chi_{1} \nabla \cdot(u \nabla w)+\mu_{1} u\left(1-u-a_{1} v\right), & x \in \Omega, t>0, \\
v_{t}=d_{2} \Delta v-\chi_{2} \nabla \cdot(v \nabla w)+\mu_{2} v\left(1-a_{2} u-v\right), & x \in \Omega, t>0, \\
-\Delta w+\lambda w=u+v, & x \in \Omega, t>0,
\end{array}\right.
$$

with the new positive parameter $\lambda$, in the physical domain $\Omega \subset \mathbb{R}^{n}, n \geqslant 1$, which we assume to be bounded with a smooth boundary $\partial \Omega$.

This system is a generalization of the classical parabolic-elliptic version of the celebrated Keller-Segel system, to the case of two species which both react to the same chemical signal (see [7,9] for details). A closely related system is studied in [3], where different diffusivities for $u$ and $v$ are allowed, $\mu_{1}=\mu_{2}=0$ and the term $\lambda w$ in the third equation is replaced with a constant. Motivated by the question whether multi-species chemotaxis mechanisms can be responsible for processes of cell sorting, the latter work focuses on the occurrence of blow-up phenomena and asymptotic behaviour of such unbounded solutions (see also $[4,5]$ for the case when $\chi_{1}$ and $\chi_{2}$ have different signs).

In contrast to this, in this work we shall concentrate on possible effects stemming from the kinetic terms in $(0.2)$, and thus address the case when both $\mu_{1}$ and $\mu_{2}$ are positive. Then if $a_{1} \geqslant 0$ and $a_{2} \geqslant 0$, the size of both $u$ and $v$ is limited by a growth restriction of logistic type. In this and related aspects, the corresponding single-species chemotaxis-growth model for $\lambda=1$

$$
\left\{\begin{array}{lll}
u_{t}=\Delta u-\chi \nabla \cdot(u \nabla w)+\mu u(1-u), & x \in \Omega, & t>0, \\
-\Delta w+w=u, & x \in \Omega, & t>0,
\end{array}\right.
$$

and its parabolic-parabolic counterpart have been studied much more thoroughly (see e.g. $[13,14,17])$. For instance, it is known that if $\mu>\left((n-2)_{+} / n\right) \chi$, then all solutions of $(0.3)$ are global and bounded [15, theorem 2.5]. Another result in this single-species framework says that when the chemotactic sensitivity is small enough as related to the logistic term in the 
sense that $2 \chi<\mu$, then the large time behaviour of any solution to the PDE system will be the same as that of the corresponding ODE; that is, we then have $u(x, t) \rightarrow 1$ and $w(x, t) \rightarrow 1$ as $t \rightarrow \infty$ [15, theorem 5.1].

It is the goal of this paper to investigate how far the latter result on global asymptotic stability of spatially uniform equilibria remains true in the two-species system (0.2). In order to formulate our results, we specify the precise mathematical setting: we shall subsequently consider $(0.2)$ along with the homogeneous Neumann boundary conditions

$$
\frac{\partial u}{\partial v}=\frac{\partial v}{\partial v}=\frac{\partial w}{\partial v}=0, \quad x \in \partial \Omega, \quad t>0,
$$

and initial conditions

$$
u(x, 0)=u_{0}(x), \quad v(x, 0)=v_{0}(x), \quad x \in \Omega .
$$

The parameters $\lambda, \chi_{1}, \chi_{2}, \mu_{1}$ and $\mu_{2}$ are assumed to be positive, and for simplicity we assume

$$
d_{1}=d_{2}=1 \text {. }
$$

Upon a straightforward computation we see that if

$$
0 \leqslant a_{1}<1 \quad \text { and } \quad 0 \leqslant a_{2}<1,
$$

then there exists a constant steady state $\left(u^{\star}, v^{\star}\right)$ with positive components given by

$$
u^{\star} \equiv \frac{1-a_{1}}{1-a_{1} a_{2}}, \quad v^{\star} \equiv \frac{1-a_{2}}{1-a_{1} a_{2}} .
$$

Our intention is to show that if, given $\mu_{1}>0$ and $\mu_{2}>0$, the cross-diffusivities $\chi_{1}$ and $\chi_{2}$ and the rates $a_{1}$ and $a_{2}$ of competitive degradation are suitably small, then all solutions of (0.2)-(0.5) will stabilize towards this equilibrium. To make this more precise, we consider the hypothesis

$$
2\left(\chi_{1}+\chi_{2}\right)+a_{1} \mu_{2}<\mu_{1} \quad \text { and } \quad 2\left(\chi_{1}+\chi_{2}\right)+a_{2} \mu_{1}<\mu_{2},
$$

and can then formulate our main result as follows.

Theorem 0.1. Let $n \geqslant 1$ and $\Omega \subset \mathbb{R}^{n}$ be a bounded domain with smooth boundary. Assume that $\lambda, \chi_{1}, \chi_{2}, \mu_{1}$ and $\mu_{2}$ are positive, that $a_{1}$ and $a_{2}$ satisfy $(0.6)$ and that $(0.8)$ holds. Then for all positive initial data $u_{0} \in C^{0}(\bar{\Omega})$ and $v_{0} \in C^{0}(\bar{\Omega})$, the solution $(u, v)$ to $(0.2)-(0.5)$ is bounded and satisfies

$$
\left\|u(\cdot, t)-u^{\star}\right\|_{L^{\infty}(\Omega)}+\left\|v(\cdot, t)-v^{\star}\right\|_{L^{\infty}(\Omega)} \rightarrow 0 \quad \text { as } t \rightarrow \infty
$$

with $u^{\star}$ and $v^{\star}$ as defined in (0.7).

We note that for any $a_{1}$ and $a_{2}$ fulfilling $(0.6)$, there exists a nonempty region $\left(\mu_{1}, \mu_{2}\right) \in \mathbb{R}_{+}^{2}$ where $(0.8)$ is satisfied.

Of course there is quite a number of possibilities of coupling between chemotaxis and other mechanisms in multi-species situations. For instance, it is conceivable that two species interact in a competitive way, but only one of them moves chemotactically. A model addressing this is considered in [10], where questions of global existence and blow-up are studied for the system

$$
\left\{\begin{array}{lll}
u_{t}=\Delta u-\nabla \cdot\left(\frac{u}{w} \nabla w\right)+u\left(1-u-a_{1} v\right), & x \in \Omega, & t>0, \\
v_{t}=\Delta v+\rho v\left(1-v-a_{2} u\right), & x \in \Omega, & t>0, \\
w_{t}=(u-\delta) w, & x \in \Omega, & t>0,
\end{array}\right.
$$

with nondiffusive evolution of the chemical. The recent work [7] which discusses a rather large class of systems related to $(0.2)$ and focuses on the existence and the use of nontrivial Lyapunov functionals. 


\section{Analysis of the associated ODE system}

In this section we analyse the ODE system

$\begin{cases}\bar{u}^{\prime}=\bar{u}\left[\mu_{1}-\left(\mu_{1}-\chi_{1}\right) \bar{u}-\chi_{1} \underline{u}+\chi_{1} \bar{v}-\left(\chi_{1}+\mu_{1} a_{1}\right) \underline{v}\right], & t>0, \\ \underline{u}^{\prime}=\underline{u}\left[\mu_{1}-\chi_{1} \bar{u}-\left(\mu_{1}-\chi_{1}\right) \underline{u}-\left(\chi_{1}+\mu_{1} a_{1}\right) \bar{v}+\chi_{1} \underline{v}\right], & t>0, \\ \bar{v}^{\prime}=\bar{v}\left[\mu_{2}+\chi_{2} \bar{u}-\left(\chi_{2}+\mu_{2} a_{2}\right) \underline{u}-\left(\mu_{2}-\chi_{2}\right) \bar{v}-\chi_{2} \underline{v}\right], & t>0, \\ \underline{v}^{\prime}\left[\mu_{2}-\left(\chi_{2}+\mu_{2} a_{2}\right) \bar{u}+\chi_{2} \underline{u}-\chi_{2} \bar{v}-\left(\mu_{2}-\chi_{2}\right) \underline{v}\right], & t>0,\end{cases}$

with initial conditions

$\bar{u}(0)=\bar{u}_{0}, \quad \underline{u}(0)=\underline{u}_{0}, \quad \bar{v}(0)=\bar{v}_{0} \quad$ and $\quad \underline{v}(0)=\underline{v}_{0}$.

The role of (1.1) with respect to (0.2)-(0.5) will become clear in the proof of lemma 2.3 in the forthcoming section.

Clearly, given any such initial data there exist $\hat{T}_{\max }=\hat{T}_{\max }\left(\bar{u}_{0}, \underline{u}_{0}, \bar{v}_{0}, \underline{v}_{0}\right) \in(0, \infty]$ and a unique solution $(\bar{u}, \underline{u}, \bar{v}, \underline{v})$ of (1.1)-(1.2) in $\left(0, \hat{T}_{\max }\right)$ which cannot be extended beyond $t=\hat{T}_{\max }$. Since $\chi_{1}$ and $\chi_{2}$ are positive, each of the equations in (1.1) contains one source term of quadratic type. It is therefore not a priori clear whether this solution is global, or finite time blow-up may occur.

On the other hand, it can easily be checked that the constant steady states of $(0.2)$ given by (0.7) determine a constant equilibrium of (1.1) in the sense that

$$
\bar{u}^{\star}=\underline{u}^{\star}=u^{\star} \equiv \frac{1-a_{1}}{1-a_{1} a_{2}}, \quad \bar{v}^{\star}=\underline{v}^{\star}=v^{\star} \equiv \frac{1-a_{2}}{1-a_{1} a_{2}}
$$

defines a nontrivial equilibrium $\left(\bar{u}^{\star}, \underline{u}^{\star}, \bar{v}^{\star}, \underline{v}^{\star}\right)$ of system (1.1). It is the goal of this section to establish the following attractivity property of this constant solution. We shall see in lemma 1.5 that whenever

$$
0<\underline{u}_{0}<\underline{u}^{*}=\bar{u}^{*}<\bar{u}_{0} \quad \text { and } \quad 0<\underline{v}_{0}<\underline{v}^{*}=\bar{v}^{*}<\bar{v}_{0},
$$

the solution of (1.1)-(1.2) will be global in time and stabilize towards $\left(\bar{u}^{\star}, \underline{u}^{\star}, \bar{v}^{\star}, \underline{v}^{\star}\right)$ in the large time limit.

To begin with, we make sure that the initially present ordering asserted by (1.4) is inherited by the solution.

Lemma 1.1. Assume (0.6) and let (1.4) hold. Then the solution of (1.1)-(1.2) satisfies

$$
0<\underline{u}(t)<\bar{u}(t) \text { and } 0<\underline{v}(t)<\bar{v}(t) \quad \text { for } \quad t \in\left(0, \hat{T}_{\max }\right) .
$$

Proof. We first observe that the positivity statements easily result from an elementary ODE comparison argument: indeed, from (1.1) we see that $\underline{u}^{\prime}=f(t) \underline{u}$ with some smooth function $f$, and hence $\underline{u}$ clearly inherits positivity of $\underline{u}_{0}$.

Thus, if $(1.5)$ was false then there would exist $t_{0} \in\left(0, \hat{T}_{\max }\right)$ such that

$$
\underline{u}<\bar{u} \quad \text { and } \underline{v}<\bar{v} \quad \text { for } t<t_{0},
$$

and such that either

$$
\underline{u}\left(t_{0}\right)=\bar{u}\left(t_{0}\right) \quad \text { and } \quad \underline{v}\left(t_{0}\right)<\bar{v}\left(t_{0}\right),
$$

or

$$
\underline{u}\left(t_{0}\right)<\bar{u}\left(t_{0}\right) \quad \text { and } \quad \underline{v}\left(t_{0}\right)=\bar{v}\left(t_{0}\right) \text {, }
$$


or

$$
\underline{u}\left(t_{0}\right)=\bar{u}\left(t_{0}\right) \quad \text { and } \quad \underline{v}\left(t_{0}\right)=\bar{v}\left(t_{0}\right) .
$$

Here we observe that if (1.9) occurred, by uniqueness of solutions to (1.1) we would evidently have $\underline{u} \equiv \bar{u}$ and $\underline{v} \equiv \bar{v}$, which contradicts (1.6).

Next, in the case (1.7) we have

$$
\frac{\bar{u}_{t}}{\overline{\bar{u}}}-\frac{\underline{u}_{t}}{\underline{u}}=\left(2 \chi_{1}-\mu_{1}\right)(\bar{u}-\underline{u})+\left(2 \chi_{1}+\mu_{1} a_{1,2}\right)(\bar{v}-\underline{v}) \quad \text { for } t>0 .
$$

By continuity of the solutions and (1.7) we would obtain

$\frac{\bar{u}_{t}}{\bar{u}}-\frac{\underline{u}_{t}}{\underline{u}}=\left(2 \chi_{1}-\mu_{1}\right)(\bar{u}-\underline{u})+\left(2 \chi_{1}+\mu_{1} a_{1,2}\right)(\bar{v}-\underline{v})>0 \quad$ for $t \in\left(t_{0}-\epsilon, t_{0}\right)$,

for $\epsilon$ small enough. An integration over $\left(t_{0}-\epsilon, t_{0}\right)$ would thus show that

$$
\log \bar{u}\left(t_{0}\right)-\log \underline{u}\left(t_{0}\right)>0
$$

and hence $\bar{u}\left(t_{0}\right)>\underline{u}\left(t_{0}\right)$, which contradicts (1.7).

Since in quite the same way we can rule out the possibility of (1.8) to occur, we thus conclude that (1.5) must be true.

Our next aim is to prove that $(\bar{u}, \underline{u}, \bar{v}, \underline{v})$ is actually global in time and bounded. This is prepared by the following.

Lemma 1.2. Let $(0.6),(0.8)$ and (1.4) hold. Then there exists $C>0$ such that the solution to (1.1)-(1.2) satisfies

$$
\bar{u} \bar{v} \leqslant C \quad \text { in }\left[0, \hat{T}_{\max }\right) .
$$

Proof. We let

$$
\varphi(t):=\ln \bar{u}(t)+\ln \bar{v}(t), \quad t \in\left[0, \hat{T}_{\max }\right) .
$$

Then using the third equation in (1.1) we compute

$$
\begin{aligned}
\varphi^{\prime}= & \frac{\bar{u}^{\prime}}{\bar{u}}+\frac{\bar{v}^{\prime}}{\bar{v}} \\
= & \mu_{1}+\mu_{2}-\left(\mu_{1}-\chi_{1}-\chi_{2}\right) \bar{u}-\left(\chi_{1}+\chi_{2}+\mu_{2} a_{2}\right) \underline{u} \\
& -\left(\mu_{2}-\chi_{1}-\chi_{2}\right) \bar{v}-\left(\chi_{1}+\chi_{2}+\mu_{1} a_{1}\right) \underline{v} \quad \text { for all } t \in\left(0, \hat{T}_{\max }\right) .
\end{aligned}
$$

Since $\bar{u}, \underline{u}, \bar{v}$ and $\underline{v}$ are nonnegative and $\chi_{1}+\chi_{2}<\min \left\{\mu_{1}, \mu_{2}\right\}$ according to $(0.8)$, we thus find $c_{1}>0$ and $c_{2}>0$ such that

$$
\varphi^{\prime} \leqslant c_{1}-c_{2}(\bar{u}+\bar{v}) \quad \text { for all } t \in\left(0, \hat{T}_{\max }\right) .
$$

Since

$$
\bar{u}+\bar{v} \geqslant 2 \sqrt{\bar{u} \bar{v}}=2 \exp (\log \sqrt{\bar{u} \bar{v}})=2 \mathrm{e}^{\frac{1}{2} \varphi},
$$

and hence (1.11) entails

$$
\varphi^{\prime}(t) \leqslant c_{1}-2 c_{2} \mathrm{e}^{\frac{1}{2} \varphi(t)} \quad \text { for all } t \in\left(0, \hat{T}_{\max }\right) .
$$

Note that $\varphi$ is a sub-solution to the equation

$$
z^{\prime}=c_{1}-2 c_{2} \mathrm{e}^{\frac{1}{2} z}, \quad z(0)=\ln \left(\bar{u}_{0} \bar{v}_{0}\right)
$$


and therefore $\varphi(t) \leqslant z(t)$ for any $t \in\left(0, \hat{T}_{\max }\right)$. There exists a unique steady states to (1.13) given by the constant $z_{*}=2 \ln \frac{c_{1}}{2 c_{2}}$. The solution $z$ tends monotonically to $z_{*}$ as $t \rightarrow \infty$. Therefore $z \leqslant \max \left\{z_{0}, z_{*}\right\}$ which implies

$$
\varphi(t) \leqslant \max \left\{\ln \bar{u}_{0}+\ln \bar{v}_{0}, 2 \ln \frac{c_{1}}{2 c_{2}}\right\} \quad \text { for all } t \in\left(0, \hat{T}_{\max }\right),
$$

and we deduce (1.10).

We can now prove global existence and boundedness of solutions to (1.1)-(1.2).

Lemma 1.3. Let (0.6), (0.8) and (1.4) be valid. Then $\hat{T}_{\max }=\infty$, and one can find $C<\infty$ such that for the solution of (1.1)-(1.2) we have

$$
\bar{u} \leqslant C \text { and } \bar{v} \leqslant C \quad \text { in }[0, \infty) .
$$

Proof. According to lemma 1.2 there exists $c_{1}>0$ such that $\bar{u} \bar{v} \leqslant c_{1}$ in $\left[0, \hat{T}_{\max }\right)$. Neglecting nonpositive terms, from the first equation in (1.1) we thus obtain

$$
\begin{aligned}
\bar{u}^{\prime} & \leqslant \mu_{1} \bar{u}-\left(\mu_{1}-\chi_{1}\right) \bar{u}^{2}+\chi_{1} \bar{u} \bar{v} \\
& \leqslant \mu_{1} \bar{u}-\left(\mu_{1}-\chi_{1}\right) \bar{u}^{2}+\chi_{1} c_{1} \quad \text { for all } t \in\left(0, \hat{T}_{\max }\right) .
\end{aligned}
$$

Upon an ODE comparison, this shows that

$$
\bar{u}(t) \leqslant \max \left\{\bar{u}_{0}, \bar{u}_{\star}\right\} \quad \text { for all } t \in\left(0, \hat{T}_{\max }\right),
$$

where

$$
\bar{u}_{\star}:=\frac{\mu_{1}+\sqrt{\mu_{1}^{2}+4 c_{1} \chi_{1}\left(\mu_{1}-\chi_{1}\right)}}{2\left(\mu_{1}-\chi_{1}\right)}
$$

is the positive steady state of the ODE $y^{\prime}=\mu_{1} y-\left(\mu_{1}-\chi_{1}\right) y^{2}+\chi_{1} c_{1}$. Along with a similar argument addressing $\bar{v}$, recalling lemma 1.1 we conclude that $(\bar{u}, \underline{u}, \bar{v}, \underline{v})$ is bounded in $\left(0, \hat{T}_{\max }\right)$. According to a standard extensibility result, this entails that in fact $\hat{T}_{\max }=\infty$, and that (1.14) holds.

The next lemma sharpens the statement from lemma 1.1.

Lemma 1.4. Assume (0.6), (0.8) and (1.4). Then the solution to (1.1)-(1.2) has the properties

$$
\bar{u}>\bar{u}^{*}=\underline{u}^{*}>\underline{u} \text { and } \bar{v}>\bar{v}^{*}=\underline{v}^{*}>\underline{v} \quad \text { in }[0, \infty),
$$

where $\left(\vec{u}^{\star}, \underline{u}^{\star}, \vec{v}^{\star}, \underline{v}^{\star}\right)$ denotes the equilibrium defined in (1.3).

Proof. Since $\left(\bar{u}^{\star}, \underline{u}^{\star}, \vec{v}^{\star}, \underline{v}^{\star}\right)$ is a constant solution of $(1.1)$, we have

$$
0=\mu_{1}-\chi_{1} \bar{u}^{*}-\left(\mu_{1}-\chi_{1}\right) \underline{u}^{*}-\left(\chi_{1}+\mu_{1} a_{1}\right) \bar{v}^{*}+\chi_{1} \underline{v}^{*} .
$$

We subtract this from the second equation in (1.1) to obtain

$\underline{u^{\prime}}=\underline{u}\left[-\chi_{1}\left(\bar{u}-\bar{u}^{*}\right)-\left(\mu_{1}-\chi_{1}\right)\left(\underline{u}-\underline{u}^{*}\right)-\left(\chi_{1}+\mu_{1} a_{1}\right)\left(\bar{v}-\bar{v}^{*}\right)+\chi_{1}\left(\underline{v}-\underline{v}^{*}\right)\right]$

Here we note that

$$
\begin{aligned}
-\chi_{1}\left(\bar{u}-\bar{u}^{*}\right)-\left(\mu_{1}-\chi_{1}\right)\left(\underline{u}-\underline{u}^{*}\right) & =-\chi_{1}(\bar{u}-\underline{u})+\chi_{1}\left(\bar{u}^{*}-\underline{u}^{*}\right)-\mu_{1}\left(\underline{u}-\underline{u}^{*}\right) \\
& =-\chi_{1}(\bar{u}-\underline{u})-\mu_{1}\left(\underline{u}-\underline{u}^{*}\right)
\end{aligned}
$$

and

$$
-\left(\chi_{1}+\mu_{1} a_{1}\right)\left(\bar{v}-\bar{v}^{*}\right)+\chi_{1}\left(\underline{v}-\underline{v}^{*}\right)=-\mu_{1} a_{1}\left(\bar{v}-\bar{v}^{*}\right)-\chi_{1}(\bar{v}-\underline{v}) .
$$


Therefore, (1.15) becomes

$$
\underline{u}^{\prime}=\underline{u}\left[-\chi_{1}(\bar{u}-\underline{u})-\mu_{1}\left(\underline{u}-\underline{u}^{*}\right)-\mu_{1} a_{1}\left(\bar{v}-\bar{v}^{*}\right)-\chi_{1}(\bar{v}-\underline{v})\right]
$$

which combined with lemma 1.1 yields the inequality

$$
\frac{\underline{u^{\prime}}}{\underline{u}}<-\mu_{1}\left(\underline{u}-\underline{u}^{*}\right)-\mu_{1} a_{1}\left(\bar{v}-\bar{v}^{*}\right) \quad \text { for all } t>0 .
$$

In the same way we see that

$$
\frac{\bar{v}^{\prime}}{\bar{v}}>-\mu_{2}\left(\bar{v}-\bar{v}^{*}\right)-\mu_{2} a_{2}\left(\underline{u}-\underline{u}^{*}\right) \quad \text { for all } t>0 .
$$

As in lemma 1.1 we proceed to argue by contradiction in proving that

$$
\underline{u}<\underline{u}^{*} \text { and } \quad \bar{v}>\bar{v}^{*} \quad \text { in }(0, \infty) .
$$

Indeed, assuming that $(1.18)$ be false we could find $t_{0} \in(0, \infty)$ such that

$$
\underline{u}<\underline{u}^{*} \quad \text { and } \quad \bar{v}>\bar{v}^{*} \quad \text { for } t<t_{0},
$$

and that either

$$
\underline{u}\left(t_{0}\right)=\underline{u}^{*} \quad \text { and } \quad \bar{v}\left(t_{0}\right)>\bar{v}^{*},
$$

or

$$
\underline{u}\left(t_{0}\right)<\underline{u}^{*} \quad \text { and } \quad \bar{v}\left(t_{0}\right)=\bar{v}^{*},
$$

or

$$
\underline{u}\left(t_{0}\right)=\underline{u}^{*} \quad \text { and } \quad \bar{v}\left(t_{0}\right)=\bar{v}^{*} .
$$

Here, if (1.20) or (1.22) holds then from (1.16) we see that $\underline{u}^{\prime}\left(t_{0}\right)<0$, which is absurd in view of (1.19). Similarly, (1.21) cannot occur because of (1.17).

We are now in the position to establish our main result on (1.1).

Lemma 1.5. Assume (0.6), (0.8) and (1.4). Then the solution of (1.1)-(1.2) satisfies

$$
\begin{array}{llll}
\bar{u}(t) \rightarrow u^{\star} & \text { and } \quad \underline{u}(t) \rightarrow u^{\star} & \text { as } t \rightarrow \infty & \text { as well as } \\
\bar{v}(t) \rightarrow v^{\star} & \text { and } \quad \underline{v}(t) \rightarrow v^{\star} & \text { as } t \rightarrow \infty, &
\end{array}
$$

where $u^{\star}$ and $v^{\star}$ are as given by (1.3).

Proof. We divide the first equation in (1.1) by $\bar{u}$ and the second by $\underline{u}$ to see upon a subtraction that is,

$$
\begin{aligned}
\frac{\mathrm{d}}{\mathrm{d} t} \log \frac{\bar{u}}{\underline{u}} & =\frac{\bar{u}_{t}}{\bar{u}}-\frac{\underline{u}_{t}}{\underline{u}} \\
& =-\left(\mu_{1}-2 \chi_{1}\right)(\bar{u}-\underline{u})+\left(2 \chi_{1}+\mu_{1} a_{1}\right)(\bar{v}-\underline{v}) .
\end{aligned}
$$

In a similar way we obtain

$$
\frac{\mathrm{d}}{\mathrm{d} t} \log \frac{\bar{v}}{\underline{v}}=\left(2 \chi_{2}+\mu_{2} a_{2}\right)(\bar{u}-\underline{u})-\left(\mu_{2}-2 \chi_{2}\right)(\bar{v}-\underline{v}) \quad \text { for all } t>0,
$$

which when added to (1.24) yields

$$
\begin{aligned}
\frac{\mathrm{d}}{\mathrm{d} t}\left(\log \frac{\bar{u}}{\underline{u}}+\log \frac{\bar{v}}{\underline{v}}\right)= & \left(-\mu_{1}+2\left(\chi_{1}+\chi_{2}\right)+\mu_{2} a_{2}\right)(\bar{u}-\underline{u}) \\
& +\left(-\mu_{2}+2\left(\chi_{1}+\chi_{2}\right)+\mu_{1} a_{1}\right)(\bar{v}-\underline{v})
\end{aligned}
$$


for all $t>0$. Here we abbreviate

$$
\varepsilon:=\min \left\{\mu_{1}-2\left(\chi_{1}+\chi_{2}\right)-\mu_{2} a_{2}, \mu_{2}-2\left(\chi_{1}+\chi_{2}\right)-\mu_{1} a_{1}\right\}
$$

and thus see that (1.25) becomes

$$
\frac{\mathrm{d}}{\mathrm{d} t}\left(\log \frac{\bar{u}}{\underline{u}}+\log \frac{\bar{v}}{\underline{v}}\right) \leqslant-\varepsilon(\bar{u}-\underline{u})-\varepsilon(\bar{v}-\underline{v}) \quad \text { for all } t>0 .
$$

Along with lemma 1.5, this first entails that

$$
\log \frac{\bar{u}}{\underline{u}} \leqslant \log \frac{\bar{u}_{0}}{\underline{u}_{0}}+\log \frac{\bar{v}_{0}}{\underline{v}_{0}}:=c_{0} \quad \text { for all } t>0,
$$

and hence using lemma 1.4 we obtain

$$
\log \frac{\bar{u}^{*}}{\underline{u}} \leqslant c_{0} \quad \text { for all } t>0 .
$$

This implies that

$$
\underline{u} \geqslant \bar{u}^{*} \mathrm{e}^{-c_{0}} \geqslant \bar{u}^{*} \frac{\underline{u}_{0}}{\bar{u}_{0}} \frac{\underline{v}_{0}}{\bar{v}_{0}}>0 \quad \text { for all } t>0 .
$$

In the same way we see that

$$
\underline{v} \geqslant \bar{v}^{*} \frac{\underline{u}_{0}}{\bar{u}_{0}} \frac{\underline{v}_{0}}{\bar{v}_{0}}>0 \quad \text { for all } t>0
$$

We now observe that by the mean value theorem, for each $t>0$ the identities

$$
\bar{u}(t)-\underline{u}(t)=\mathrm{e}^{\xi_{1}(t)}(\log \bar{u}(t)-\log \underline{u}(t))
$$

and

$$
\bar{v}(t)-\underline{v}(t)=\mathrm{e}^{\xi_{2}(t)}(\log \bar{v}(t)-\log \underline{v}(t))
$$

hold with some $\xi_{1}(t) \in(\log \underline{u}(t), \log \bar{u}(t))$ and $\xi_{2}(t) \in(\log \underline{v}(t), \log \bar{v}(t))$. Hence by $(1.27)$ and (1.28), from (1.26) we infer that

$$
\frac{\mathrm{d}}{\mathrm{d} t}\left(\log \frac{\bar{u}}{\underline{u}}+\log \frac{\bar{v}}{\underline{v}}\right) \leqslant-\varepsilon_{0}\left(\log \frac{\bar{u}}{\underline{u}}+\log \frac{\bar{v}}{\underline{v}}\right) \quad \text { for all } t>0
$$

is valid with

$$
\varepsilon_{0}=\varepsilon \frac{\underline{u}_{0}}{\overline{\bar{u}}_{0}} \frac{\underline{v}_{0}}{\bar{v}_{0}} \min \left\{\bar{u}^{*}, \bar{v}^{*}\right\} .
$$

Therefore an ODE comparison shows that

$$
\log \frac{\bar{u}}{\underline{u}}+\log \frac{\bar{v}}{\underline{v}} \leqslant\left(\log \frac{\bar{u}_{0}}{\underline{u}_{0}}+\log \frac{\bar{v}_{0}}{\underline{v}_{0}}\right) \mathrm{e}^{-\varepsilon_{0} t} \quad \text { for all } t>0 .
$$

According to lemma 1.5, this entails the inequalities

$$
0<\log \frac{\bar{u}}{\underline{u}} \leqslant\left(\log \frac{\bar{u}_{0}}{\underline{u}_{0}}+\log \frac{\bar{v}_{0}}{\underline{v}_{0}}\right) \mathrm{e}^{-\varepsilon_{0} t} \quad \text { for all } t>0
$$

and

$$
0<\log \frac{\bar{v}}{\underline{v}} \leqslant\left(\log \frac{\bar{u}_{0}}{\underline{u}_{0}}+\log \frac{\bar{v}_{0}}{\underline{v}_{0}}\right) \mathrm{e}^{-\varepsilon_{0} t} \quad \text { for all } t>0
$$

and thereby shows that

$$
|\bar{u}(t)-\underline{u}(t)|+|\bar{v}(t)-\underline{v}(t)| \rightarrow 0 \quad \text { as } t \rightarrow \infty .
$$

In view of lemma 1.4 this proves (1.23). 


\section{Relating the PDE system to the ODE system. Proof of theorem 0.1}

We now turn our attention to the original problem $(0.2)-(0.5)$. As a preliminary, we state the following result on local existence and uniqueness of solutions which can be proved by a straightforward adaptation of well-established methods (see [15, theorem 2.1], [1, section 1], for instance).

Lemma 2.1. Let $\lambda, \chi_{1}, \chi_{2}, \mu_{1}$ and $\mu_{2}$ be positive and $a_{1} \geqslant 0$ as well as $a_{2} \geqslant 0$. Suppose that $u_{0}$ and $v_{0}$ belong to $C^{0}(\bar{\Omega})$ and are nonnegative. Then there exist $T_{\max } \in(0, \infty]$ and a unique triple $(u, v, w)$ of nonnegative functions belonging to $C^{0}\left(\bar{\Omega} \times\left[0, T_{\max }\right)\right) \cap C^{2,1}\left(\bar{\Omega} \times\left(0, T_{\max }\right)\right)$ which solve (0.2)-(0.5) in the classical sense in $\Omega \times\left(0, T_{\max }\right)$. Moreover,

either $T_{\max }=\infty \quad$ or $\quad\|u(\cdot, t)\|_{L^{\infty}(\Omega)}+\|v(\cdot, t)\|_{L^{\infty}(\Omega)} \rightarrow \infty \quad$ as $t \nearrow T_{\max }$.

According to the strong maximum principle applied to the first two equations in $(0.2)$, replacing $t$ by $t+\tau$ for sufficiently small $\tau>0$ we may assume that

$$
u_{0}>0 \text { and } v_{0}>0 \text { in } \bar{\Omega} .
$$

It is therefore possible to find positive numbers $\bar{u}_{0}, \underline{u}_{0}, \bar{v}_{0}$ and $\underline{v}_{0}$ such that the inequalities in (1.4) hold as well as

$$
\underline{u}_{0} \leqslant u_{0}(x) \leqslant \bar{u}_{0} \quad \text { and } \quad \underline{v}_{0} \leqslant v_{0}(x) \leqslant \bar{v}_{0} \quad \text { for all } x \in \Omega .
$$

We now let $(\bar{u}, \underline{u}, \bar{v}, \underline{v})$ denote the solution of (1.1)-(1.2) corresponding to these fixed initial data, and plan to prove that under the assumptions $(0.8)$ and $(0.6)$, the inequalities $\underline{u} \leqslant u \leqslant \bar{u}$ and $\underline{v} \leqslant v \leqslant \bar{v}$ will remain valid throughout $\Omega \times\left(0, T_{\max }\right)$. To this end, in lemma 2.3 we shall derive an appropriate differential inequality for some functional involving the functions $\bar{U}, \underline{U}$, $\bar{V}$ and $\underline{V}$ which are defined by setting

$$
\bar{U}(x, t):=u(x, t)-\bar{u}(t), \quad \underline{U}(x, t):=u(x, t)-\underline{u}(t),
$$

and

$$
\bar{V}(x, t):=v(x, t)-\bar{v}(t), \quad \underline{V}(x, t):=v(x, t)-\underline{v}(t)
$$

for $(x, t) \in \Omega \times\left[0, T_{\max }\right)$, in order to verify that the positive parts $\bar{U}_{+}$and $\bar{V}_{+}$and the negative parts $\underline{U}$ and $\underline{V}$ - are negative throughout $\Omega \times\left(0, T_{\max }\right)$. As a preparation for this, we state the following.

Lemma 2.2. With $\bar{u}, \underline{u}, \bar{v}$ and $\underline{v}$ as defined above, the solution of $(0.2)-(0.5)$ has the properties

$$
\int_{\Omega}(\lambda w-\bar{u}-\bar{v})_{+}^{2} \leqslant 2 \int_{\Omega}\left(\bar{U}_{+}^{2}+\bar{V}_{+}^{2}\right) \quad \text { for all } t \in\left(0, T_{\max }\right)
$$

and

$$
\int_{\Omega}(\lambda w-\underline{u}-\underline{v})_{-}^{2} \leqslant 2 \int_{\Omega}\left(\underline{U}_{-}^{2}+\underline{V}_{-}^{2}\right) \quad \text { for all } t \in\left(0, T_{\max }\right) .
$$

Proof. From the third equation in (1.1) we have

$$
-\Delta w+\lambda w-\bar{u}-\bar{v}=\bar{U}+\bar{V} \quad \text { in } \Omega \times\left(0, T_{\max }\right),
$$

and after multiplying this by $(\lambda w-\bar{u}-\bar{v})_{+}$and integrating over $\Omega$ we obtain

$\frac{1}{\lambda} \int_{\Omega}\left|\nabla(\lambda w-\bar{u}-\bar{v})_{+}\right|^{2}+\int_{\Omega}(\lambda w-\bar{u}-\bar{v})_{+}^{2}=\int_{\Omega}(\bar{U}+\bar{V})(\lambda w-\bar{u}-\bar{v})_{+}$

for all $t \in\left(0, T_{\max }\right)$. Here we apply Young's inequality to see that

$$
\int_{\Omega}(\bar{U}+\bar{V})(\lambda w-\bar{u}-\bar{v})_{+} \leqslant \frac{1}{2} \int_{\Omega}(\bar{U}+\bar{V})_{+}^{2}+\frac{1}{2} \int_{\Omega}(\lambda w-\bar{u}-\bar{v})_{+}^{2} .
$$


Since

$$
(\bar{U}+\bar{V})_{+}^{2} \leqslant\left(\bar{U}_{+}+\bar{V}_{+}\right)^{2} \leqslant 2 \bar{U}_{+}^{2}+2 \bar{V}_{+}^{2},
$$

from (2.8) we thus find

$\frac{1}{\lambda} \int_{\Omega}\left|\nabla(\lambda w-\bar{u}-\bar{v})_{+}\right|^{2}+\frac{1}{2} \int_{\Omega}(\lambda w-\bar{u}-\bar{v})_{+}^{2} \leqslant \int_{\Omega} \bar{U}_{+}^{2}+\bar{V}_{+}^{2} \quad$ for all $t \in\left(0, T_{\max }\right)$, which implies (2.5). Inequality (2.6) can be seen in an analogous way.

We can now prove the announced two-sided pointwise estimates for the solution of $(0.2)-$ (0.5).

Lemma 2.3. The solution of (0.2)-(0.5) satisfies

$$
\underline{u}(t) \leqslant u(x, t) \leqslant \bar{u}(t) \quad \text { for all } x \in \Omega \text { and } t \in\left(0, T_{\max }\right)
$$

and

$$
\underline{v}(t) \leqslant v(x, t) \leqslant \bar{v}(t) \quad \text { for all } x \in \Omega \text { and } t \in\left(0, T_{\max }\right),
$$

where $\underline{u}, \bar{u}, \underline{v}$ and $\bar{v}$ are as specified above.

Proof. We claim that for each $T \in\left(0, T_{\max }\right)$ we have

$$
\underline{u}(t) \leqslant u(x, t) \leqslant \bar{u}(t) \quad \text { for all } x \in \Omega \text { and } t \in(0, T)
$$

and

$$
\underline{v}(t) \leqslant v(x, t) \leqslant \bar{v}(t) \quad \text { for all } x \in \Omega \text { and } t \in(0, T) .
$$

To verify this, we first observe that given any such $T \in\left(0, T_{\max }\right)$, since $(u, v, w)$ is smooth in $\bar{\Omega} \times[0, T]$ we can find $c_{1}(T)>0$ such that

$u(x, t) \leqslant c_{1}(T), \quad v(x, t) \leqslant c_{1}(T) \quad$ and $\quad w(x, t) \leqslant c_{1}(T)$

for all $x \in \Omega$ and $t \in(0, T)$, where in view of lemmas 1.1 and 1.3 we may assume upon enlarging $c_{1}(T)$ if necessary that in addition

$\bar{u}(t) \leqslant c_{1}(T), \quad \underline{u}(t) \leqslant c_{1}(T), \quad \bar{v}(t) \leqslant c_{1}(T) \quad$ and $\quad \underline{v}(t) \leqslant c_{1}(T) \quad$ for $t \in(0, T)$.

Now from the first and the third equations in $(0.2)$ we obtain

$$
u_{t}-\Delta u+\chi_{1} \nabla u \nabla w=\chi_{1} u(u+v-\lambda w)+\mu_{1} u\left(1-u-a_{1} v\right),
$$

in $x \in \Omega, t \in\left(0, T_{\max }\right)$, from which we subtract the first ODE in (1.1) to see that $\bar{U}=u-\bar{u}$ satisfies

$$
\begin{aligned}
\bar{U}_{t}-\Delta \bar{U}+\chi_{1} \nabla \bar{U} \cdot \nabla w= & \chi_{1} u(u+v-\lambda w)+\mu_{1} u\left(1-u-a_{1} v\right) \\
& -\bar{u} \cdot\left[\mu_{1}-\left(\mu_{1}-\chi_{1}\right) \bar{u}-\chi_{1} \underline{u}-\left(\chi_{1}-\mu_{1} a_{1}\right) \underline{v}\right] \\
= & \mu_{1} \bar{U}+u\left[\left(\chi_{1}-\mu_{1}\right) u+\left(\chi_{1}-\mu_{1} a_{1}\right) v-\lambda \chi_{1} w\right] \\
& -\bar{u} \cdot\left[\left(\chi_{1}-\mu_{1}\right) \bar{u}-\chi_{1} \underline{u}+\chi_{1} \bar{v}-\left(\chi_{1}+\mu_{1} a_{1}\right) \underline{v}\right]
\end{aligned}
$$

in $\Omega \times\left(0, T_{\max }\right)$. Here we express the kinetic part in terms of the functions $\bar{U}, \bar{V}=v-\bar{v}$ and $\underline{V}=v-\underline{v}$ upon observing that

$$
u\left(\chi_{1}-\mu_{1}\right) u-\bar{u}\left(\chi_{1}-\mu_{1}\right) \bar{u}=\left(\chi_{1}-\mu_{1}\right)(u+\bar{u}) \bar{U}
$$

and

$$
\left.u\left(\chi_{1}-\mu_{1} a_{1,2}\right) v-\bar{u}\left(\chi_{1} \bar{v}-\mu_{1} a_{1} \underline{v}\right)\right)=\chi_{1}(v \bar{U}+\bar{u} \bar{V})-\mu_{1} a_{1}(v \bar{U}+\bar{u} \underline{V})
$$


as well as

$$
-\chi_{1} \lambda u w+\chi_{1} \bar{u}(\underline{u}+\underline{v})=-\chi_{1} \lambda w \bar{U}+\chi_{1} \bar{u}(\underline{u}+\underline{v}-\lambda w) .
$$

From (2.14) we thereby obtain the identity

$$
\begin{aligned}
\bar{U}_{t}-\Delta \bar{U}+\chi_{1} \nabla \bar{U} \nabla w= & \bar{U}\left[\mu_{1}+\left(\chi_{1}-\mu_{1}\right)(u+\bar{u})+\left(\chi_{1}-\mu_{1} a_{1}\right) v-\chi_{1} \lambda w\right] \\
& +\chi_{1} \bar{u} \bar{V}-\mu_{1} a_{1} \bar{u} \underline{V}+\chi_{1}(\underline{u}+\underline{v}-\lambda w) .
\end{aligned}
$$

We multiply this by $\bar{U}_{+}$and integrate by parts over $\Omega$ to obtain, writing

$$
b(x, t):=\mu_{1}+\left(\chi_{1}-\mu_{1}\right)(u+\bar{u})+\left(\chi_{1}-\mu_{1} a_{1}\right) v-\chi_{1} \lambda w,
$$

that

$$
\begin{aligned}
\frac{\mathrm{d}}{\mathrm{d} t} \frac{1}{2} \int_{\Omega} \bar{U}_{+}^{2}+\int_{\Omega}\left|\nabla \bar{U}_{+}\right|^{2}= & -\frac{\chi_{1}}{2} \int_{\Omega} \nabla \bar{U}_{+}^{2} \nabla w+\int_{\Omega} b(x, t) \bar{U}_{+}^{2} \\
& +\chi_{1} \int_{\Omega} \bar{u} \overline{V U}_{+}-\mu_{1} a_{1} \int_{\Omega} \bar{u} \underline{\underline{U}} \bar{U}_{+}+\chi_{1} \int_{\Omega}(\underline{u}+\underline{v}-\lambda w) \bar{U}_{+}
\end{aligned}
$$

holds for all $t \in\left(0, T_{\max }\right)$. Here another integration by parts along with the third equation in (0.2) shows that

$$
-\frac{\chi_{1}}{2} \int_{\Omega} \nabla \bar{U}_{+}^{2} \cdot \nabla w=\frac{\chi_{1}}{2} \int_{\Omega} \bar{U}_{+}^{2} \Delta w=-\frac{\chi_{1}}{2} \int_{\Omega} \bar{U}_{+}^{2}(u+v-\lambda w) .
$$

Thanks to (2.13), we can estimate

$$
|u+v-\lambda w| \leqslant(2+\lambda) c_{1}(T) \quad \text { in } \Omega \times(0, T),
$$

so that (2.17) implies that

$$
-\frac{\chi_{1}}{2} \int_{\Omega} \nabla \bar{U}_{+}^{2} \cdot \nabla w \leqslant \frac{(2+\lambda) \chi_{1} c_{1}(T)}{2} \int_{\Omega} \bar{U}_{+}^{2} \quad \text { for } t \in(0, T) .
$$

By means of Young's inequality, the rightmost three integrals in (2.16) can be estimated according to

and

$$
\chi_{1} \int_{\Omega} \bar{u} \overline{V U}_{+} \leqslant \chi_{1} \int_{\Omega} \bar{u} \bar{V}_{+} \bar{U}_{+} \leqslant \frac{\chi c_{1}(T)}{2}\left(\int_{\Omega} \bar{V}_{+}^{2}+\int_{\Omega} \bar{U}_{+}^{2}\right)
$$

$$
-\mu_{1} a_{1} \int_{\Omega} \bar{u} \underline{\underline{V}} \bar{U}_{+} \leqslant \mu_{1} a_{1} \int_{\Omega} \bar{u} \underline{V} \underline{U}_{+} \leqslant \frac{\mu_{1} a_{1} c_{1}(T)}{2}\left(\int_{\Omega} \underline{V}_{-}^{2}+\int_{\Omega} \bar{U}_{+}^{2}\right)
$$

as well as

$\chi_{1} \int_{\Omega}(\underline{u}+\underline{v}-\lambda w) \bar{U}_{+} \leqslant \chi_{1} \int_{\Omega}(\underline{u}+\underline{v}-\lambda w)_{+} \bar{U}_{+} \leqslant \frac{\chi_{1}}{2}\left(\int_{\Omega}(\underline{u}+\underline{v}-\lambda w)_{+}^{2}+\bar{U}_{+}^{2}\right)$

for all $t \in(0, T)$. Recalling lemma 2.2 we thus see that

$$
\chi_{1} \int_{\Omega}(\underline{u}+\underline{v}-\lambda w) \bar{U}_{+} \leqslant \frac{\chi_{1}}{2}\left(2 \int_{\Omega} \underline{U}_{-}^{2}+2 \int_{\Omega} \underline{V}_{-}^{2}+\int_{\Omega} \bar{U}_{+}^{2}\right)
$$

for $t \in(0, T)$. In light of (2.18)-(2.21) and the fact that

$$
|b(x, t)| \leqslant c_{2}(T):=\mu_{1}+\left[(3+\lambda) \chi_{1}+\left(2+a_{1}\right) \mu_{1}\right] c_{1}(T)
$$

for all $x \in \Omega$ and $t \in(0, T),(2.16)$ entails that

$$
\frac{\mathrm{d}}{\mathrm{d} t} \frac{1}{2} \int_{\Omega} \bar{U}_{+}^{2}+\int_{\Omega}\left|\nabla \bar{U}_{+}\right|^{2} \leqslant k_{1}(T)\left(\int_{\Omega} \bar{U}_{+}^{2}+\int_{\Omega} \underline{U}_{-}^{2}+\int_{\Omega} \bar{V}_{+}^{2}+\int_{\Omega} \underline{V}_{-}^{2}\right)
$$


for all $t \in(0, T)$, where $k_{1}(T)$ is defined by

$k_{1}(T):=\max \left\{\frac{(2+\lambda) \chi_{1} c_{1}(T)}{2}+c_{2}(T)+\frac{\chi_{1} c_{1}(T)}{2}+\frac{\mu_{1} a_{1} c_{1}(T)}{2}+\frac{\chi_{1}}{2}, \frac{\mu_{1} a_{1} c_{1}(T)}{2}+\chi_{1}\right\}$.

In the same fashion as before we multiply the equation satisfied by $\underline{U}$ by $\underline{U}$ - to derive, using similar computations as those in (2.18)-(2.21), the inequality

$\frac{\mathrm{d}}{\mathrm{d} t} \frac{1}{2} \int_{\Omega} \underline{U}_{-}^{2}+\int_{\Omega}|\nabla \underline{U}-|^{2} \leqslant k_{2}(T)\left(\int_{\Omega} \bar{U}_{+}^{2}+\int_{\Omega} \underline{U}^{2}+\int_{\Omega} \bar{V}_{+}^{2}+\int_{\Omega} \underline{V}^{2}\right)$

for all $t \in(0, T)$ with certain positive constants $k_{2}$. By symmetry we obtain a constants $k_{3}(T)$ and $k_{4}(T)$ such that

$\frac{\mathrm{d}}{\mathrm{d} t} \frac{1}{2} \int_{\Omega} \bar{V}_{+}^{2}+\int_{\Omega}\left|\nabla \bar{V}_{+}\right|^{2} \leqslant k_{3}(T)\left(\int_{\Omega} \bar{U}_{+}^{2}+\int_{\Omega} \underline{U}_{-}^{2}+\int_{\Omega} \bar{V}_{+}^{2}+\int_{\Omega} \underline{V}_{-}^{2}\right)$

and

$\frac{\mathrm{d}}{\mathrm{d} t} \frac{1}{2} \int_{\Omega} \underline{V}_{-}^{2}+\int_{\Omega} \mid \nabla \underline{V} \underline{-}_{-}^{2} \leqslant k_{4}(T)\left(\int_{\Omega} \bar{U}_{+}^{2}+\int_{\Omega} \underline{U}_{-}^{2}+\int_{\Omega} \bar{V}_{+}^{2}+\int_{\Omega} \underline{V}_{-}^{2}\right)$

for all $t \in(0, T)$. We finally add (2.23), (2.24), (2.25) and (2.26) to see that

$$
\frac{\mathrm{d}}{\mathrm{d} t} \int_{\Omega}\left(\bar{U}_{+}^{2}+\underline{U}^{2}+\bar{V}_{+}^{2}+\underline{V}_{+}^{2}\right) \leqslant k(T) \int_{\Omega}\left(\bar{U}_{+}^{2}+\underline{U}_{-}^{2}+\bar{V}_{+}^{2}+\underline{V}^{2}\right)
$$

for $t \in(0, T)$ and $k(T):=\max _{i=1 \ldots 4}\left\{k_{i}(T)\right\}$. Since $\bar{U}_{+}=\underline{U}_{-}=\bar{V}_{+}=\underline{V}_{-}=0$ at $t=0$ according to our restriction (2.2) on $\bar{u}_{0}, \underline{u}_{0}, \bar{v}_{0}$ and $\underline{v}_{0}$, an ODE comparison shows that

$$
\int_{\Omega}\left(\bar{U}_{+}^{2}+\underline{U}_{-}^{2}+\bar{V}_{+}^{2}+\underline{V}_{+}^{2}\right)=0 \quad \text { for all } t \in(0, T),
$$

which in view of definitions (2.3) and (2.4) yields (2.11) and (2.12). Since $T \in\left(0, T_{\max }\right)$ was arbitrary, this proves the lemma.

Now our main results are an immediate consequence.

Proof of theorem 0.1. In view of (2.1), inequalities (2.9) and (2.10) rule out the possibility that $T_{\max }$ be finite. Therefore, the orderings asserted by lemma 2.3 are actually valid in the entire parabolic cylinder $\Omega \times(0, \infty)$, and hence lemma 1.5 entails that $(0.9)$ holds.

\section{Conclusion and discussion}

We have considered a model for the spatio-temporal evolution of two biological species which are attracted by the same chemical stimulus, and which proliferate and compete for the same resources. We have seen that the unique nontrivial spatially homogeneous steady state of the system, as given by $(0.7)$, is globally asymptotically stable under the hypotheses $(0.8)$ which essentially reflect the assumption that the effects stemming from chemotactic cross-diffusion and competitive degradation are sufficiently small.

This study is to be understood as a first step towards understanding possible effects of chemotactic interaction on Lotka-Volterra-type competitive evolution of motile cell populations. An interesting open question is in how far assumptions complementary to ours may go along with types of solution behaviour that reflect spontaneous spatial self-organization and thus may be interpreted as processes of cell sorting.

Addressing the mathematically most extreme formulation thereof, one might pursue the question whether blow-up phenomena may occur in the considered setting. In light of 
known results for the corresponding borderline case, it seems natural to conjecture that such a singularity formation might be possible under appropriate smallness assumptions on the parameters $\mu_{1}$ and $\mu_{2}$ which measure the strength of the kinetic terms. Here the literature on the corresponding single-species analogues suggest to believe that it is conceivable that the occurrence of exploding solutions is ruled out is enhanced by large space dimensions, but that considerable mathematical obstacles, arising even in the single-species framework, are to be expected (see $[15,18]$, for instance).

Less drastic interpretations of spatial structuring include bounded solutions which however do not stabilize towards homogeneous profiles. Here deeper insight can be expected upon addressing the corresponding basic mathematical issues of (nonlinear) instability of constant steady states, or existence of nonconstant equlibria. But these require entirely different approaches than pursued here, and thus need to be discussed elsewhere.

\section{References}

[1] Cieślak T and Winkler M 2008 Finite-time blow-up in a quasilinear system of chemotaxis Nonlinearity 21 1057-76

[2] Delgado M, Morales-Rodrigo C, Suárez A and Tello J I 2010 On a parabolic-elliptic chemotactic model with coupled boundary conditions Nonlinear Anal.: Real World Appl. $113884-902$

[3] Espejo-Arenas E E, Stevens A and Velázquez J J L 2009 Simultaneous finite time blow-up in a two-species model for chemotaxis Analysis $29317-38$

[4] Espejo-Arenas EE, Stevens A and Velázquez J J L 2010 A note on non-simultaneous blow-up for a drift-diffusion model Diff. Integral Eqns. 23 451-62

[5] Espejo-Arenas E E, Stevens A and Suzuki T 2011 Simultaneous blowup and mass separation during collapse in an interacting system of chemotactic species, preprint 64/201 1, Max-Planck Institut, http://www.mis.mpg.de/ publications/preprints/2011

[6] Hillen T and Painter K J 2009 A user's guide to PDE models for chemotaxis J. Math. Biol. 58 183-217

[7] Horstmann D 2011 Generalizing the Keller-Segel model: Lyapunov functionals, steady state analysis, and blow-up results for multi-species chemotaxis models in the presence of attraction and repulsion between competitive interacting species J. Nonlinear Sci. 21 231-70

[8] Jäger W and Luckhaus S 1992 On explosions of solutions to a system of partial differential equations modelling chemotaxis Trans. Am. Math. Soc. 329 819-24

[9] Keller E F and Segel L A 1970 Initiation of slime mold aggregation viewed as an instability J. Theor: Biol. $26399-415$

[10] Li J F and Liu W A 2010 Existence and behavior of solution to some chemotaxis model with interacting species Int. J. Biomath. 3 367-82

[11] Mimura M and Tsujikawa T 1996 Aggregation pattern dynamics in a chemotaxis model including growth Physica A $230499-543$

[12] Murray J D 1993 Mathematical Biology (Berlin: Springer)

[13] Osaki K, Tsujikawa T, Yagi A and Mimura M 2002 Exponential attracktor for a chemotaxis-growth system of equations Nonlinear Anal.: Theory Methods Appl. 51 119-44

[14] Osaki K and Yagi A 2002 Global existence for a chemotaxis-growth system in $\mathbb{R}^{2}$ Adv. Math. Sci. Appl. $12587-606$

[15] Tello J I and Winkler M 2007 A chemotaxis system with logistic source Commun. Partial Diff. Eqns 32 $849-77$

[16] Weijer C 2004 Dictyostelium morphogenesis Curr. Opin. Genet. Dev. 14 392-8

[17] Winkler M 2010 Boundedness in the higher-dimensional parabolic-parabolic chemotaxis system with logistic source Commun. Partial Diff. Eqns. 35 1516-37

[18] Winkler M 2011 Blow-up in a higher-dimensional chemotaxis system despite logistic growth restriction J. Math. Anal. Appl. 384 261-72 